\title{
Nuevos registros del Oso Andino Tremarctos ornatus (Carnivora: Ursidae) en Cerro Pintao, Serranía del Perijá, sur de La Guajira, Colombia
}

\author{
Roger Ayazo-Bi ${ }^{1 *}$, Víctor Flórez-Carrillo², Leidys Murillo-Ramos³id, Larry Jiménez- \\ Ferbans ${ }^{4}$ id \\ 1 Grupo de investigación "Biodiversidad-Unicórdoba", Departamento de Biología, Universidad de Córdoba, Montería, \\ Colombia \\ 2 Cerro Pintao, Villanueva, La Guajira, Colombia \\ 3 Grupo de investigación "Biología Evolutiva”, Departamento de Biología y Química, Universidad de Sucre, Sincelejo, Colombia \\ 4 Grupo de investigación "Evolución, Sistemática y Ecología Molecular", Universidad del Magdalena, Santa Marta, Colombia \\ *Correspondencia: ayazob@gmail.com
}

\section{Resumen}

En esta nota se reporta un nuevo registro de campo de la presencia de Tremarctos ornatus en Urumita, sur de la Guajira, frontera con Venezuela. El reporte está representado por el hallazgo de heces frescas en un sector boscoso del Cerro Pintao. Adicionalmente, documentamos algunos registros anecdóticos de avistamientos de la especie por pobladores locales en el municipio de Villanueva. Estos avistamientos se hicieron fuera de los límites del Parque Natural Regional Cerro Pintao.

Palabras clave: Andes colombianos, bosque nublado, Oso de anteojos.

\begin{abstract}
This note reports a new record of the presence of Tremarctos ornatus in Urumita, south of La Guajira, at the border with Venezuela. The report is represented by the finding of fresh feces in a forest sector of Cerro Pintao. Additionally, we documented some anecdotal records of sightings of the species by local residents in the municipality of Villanueva. These sightings were made outside the limits of the Cerro Pintao Regional Natural Park.
\end{abstract}

Key words: Colombian Andes, cloud forest, Spectacled bear.

El Oso Andino (Tremarctos ornatus Cuvier 1825) es una especie endémica de los Andes tropicales, se distribuye desde Bolivia hasta Venezuela donde habita desde tierras bajas hasta páramos por encima de los 4.000 m, siendo más común en bosques nublados (Kattan et al. 2004). En Colombia, T. ornatus habita en los tres ramales de los Andes, y aunque desde 1973 ha sido categorizado como "Vulnerable" por la UICN (García-Rangel 2012; VelezLiendo \& García-Rangel 2017), los esfuerzos encaminados al conocimiento de la historia natural, actualización de la distribución y estrategias de conservación de esta especie en 
el país han sido insuficientes (Rodríguez-Castro et al. 2015). La Serranía del Perijá, región natural que limita con Venezuela, es reconocida como una de las zonas donde habita la especie. Sin embargo, en la vertiente colombiana no existía confirmación de la presencia de $T$. ornatus, siendo los registros más antiguos referidos del lado venezolano (Mondolfi 1989; Viloria et al. 1995). Recientemente Rodríguez y colaboradores (2019) publicaron registros de esta especie provenientes de expediciones realizadas entre 2009 y 2016 en los departamentos de Cesar y La Guajira así como algunos registros extraídos de revisión de literatura científica (Rodríguez et al. 2011; 2014; 2016).

En esta nota reportamos, después de nueve años, un nuevo registro de campo de la presencia de T. ornatus en Urumita, sur de la Guajira (ver Rodríguez et al. 2019). El reporte está representado por el hallazgo de heces frescas en un sector boscoso del Cerro Pintao. Adicionalmente, documentamos algunos registros anecdóticos de avistamientos de la especie por pobladores locales de Villanueva.

A mediados de enero de 2019, durante una expedición de cuatro días a la zona montañosa de Urumita (sur de La Guajira), se detectaron dos excretas frescas en uno de los senderos del Cerro Pintao, Serranía del Perijá (Figura 1a) (10,45972, -72,86879, WGS84; 2.800 msnm). Las excretas, que tenían una dimensión de $15,6 \mathrm{~cm}$ de ancho por 20,8 cm de largo y 20,8 $\mathrm{cm}$ de ancho por 31,2 cm de largo, respectivamente, se encontraban al lado del sendero y presentaban una coloración marrón y consistencia pastosa (Figura 1b). Una inspección meticulosa reveló que estaban compuestas por semillas de una especie de la familia Arecaceae (2,7 cm de largo por 1,5 cm de ancho).

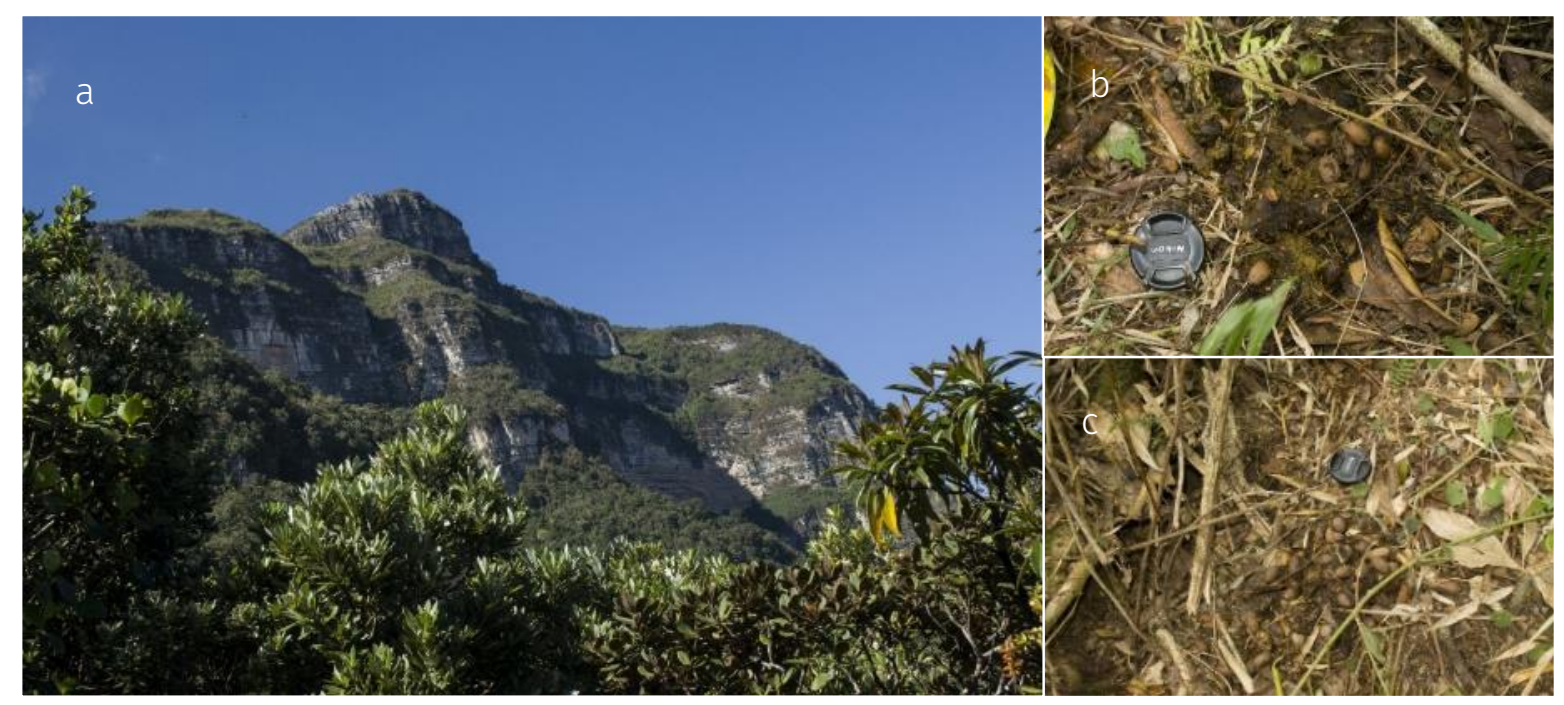

FIGURA 1. Vista del Cerro Pintao, Serranía del Perijá, sur de La Guajira (a). Excretas de Oso Andino caracterizadas por abundantes semillas de una palma (Arecaceae). Como referencia de tamaño se usó la tapa protectora de un lente Nikon 18-55 mm (b y c).

La anterior evidencia sugiere que estas excretas pertenecen a un Oso Andino, el mamífero más grande que tiene distribución en esta zona y cuya dieta omnivora incluye, entre otras, plantas y frutos de bromelias, lauráceas y arecáceas (Cavelier et al. 2010). Es característico que las heces de T. ornatus contengan semillas enteras no depredadas, por lo que se considera a esta especie como un dispersor de semillas (García-Rangel 2012). La distancia entre las excretas no fue mayor a 300 m lineales, por lo que podrían pertenecer al mismo individuo. La zona del hallazgo se caracterizó por presentar una capa de aproximadamente 
$30 \mathrm{~cm}$ de hojarasca y raíces de árboles, y alta abundancia de epífitas en árboles y a nivel del suelo.

Por otra parte, en 2016 en la finca "La Culebrera" se avistó un individuo a una distancia inferior a 50 m. En junio de 2018, en el sector "La Montaña" (10,51245, -72,89521, WGS84) y en "Las Mezas" (10,524650, -72,926636, WGS84) se observó un Oso Andino en los alrededores de las viviendas (Figura 2).

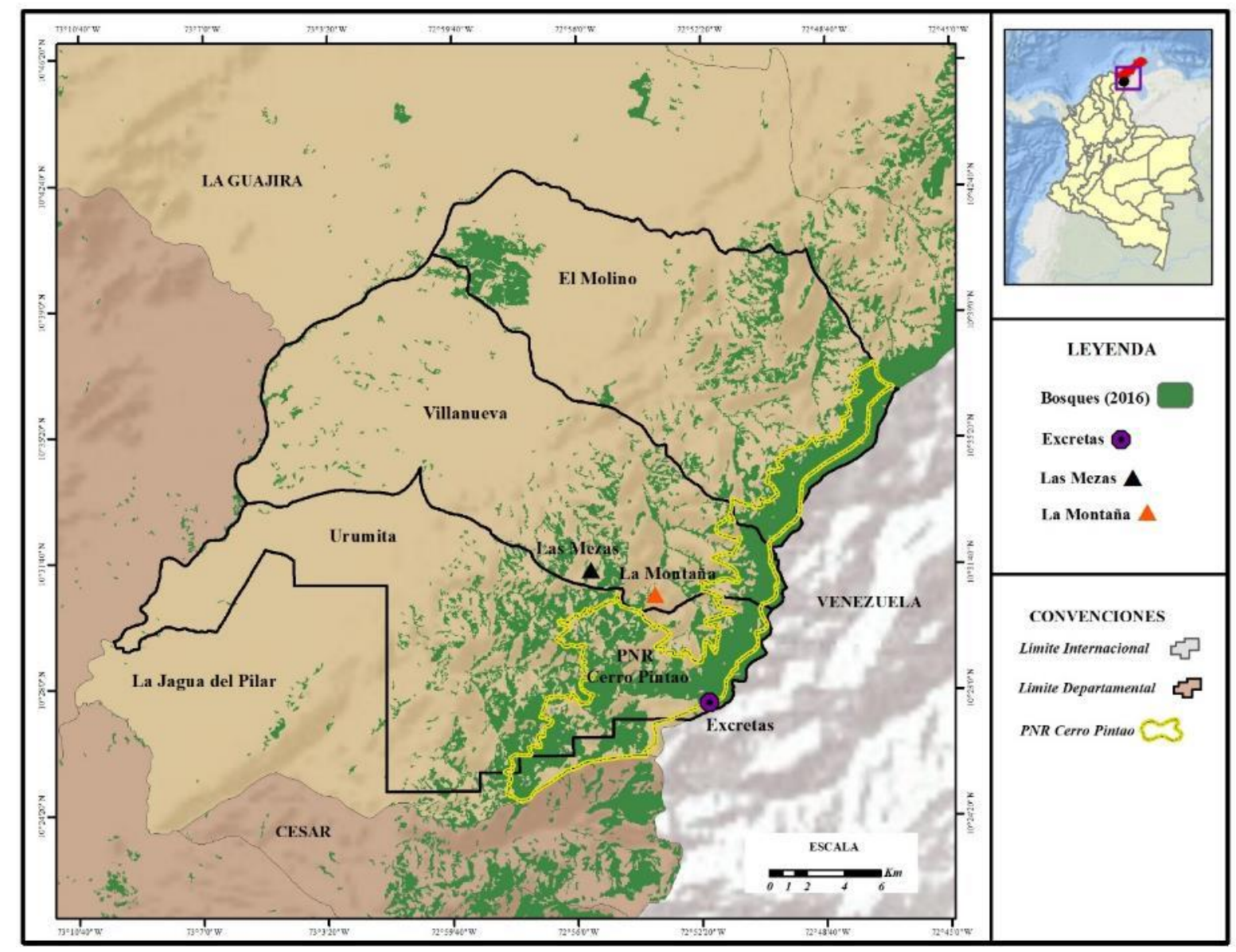

FIGURA 2. Registros de la presencia del Oso Andino (T. ornatus) en el Cerro Pintao, Serranía del Perijá, sur de La Guajira, Colombia. Triángulos: avistamientos por pobladores locales (ubicados fuera del área protegida), punto sólido: excretas.

En 2019 se registraron otros avistamientos en la finca "Estado Falcón”, donde se observó un Oso Andino alimentándose de los frutos de un árbol; en la Sierra Negra, finca "Los Estados", "Las Flores" y el sector "La Popa". Igualmente se han hecho avistamientos en la finca "El Cairo" y "Los Llanos". Lo anterior sugiere que esta especie usa zonas de esta región.

Al igual que Rodríguez et al. (2019), la evidencia reportada en esta nota señala que T. ornatus usa los bosques de la parte montañosa del sur de La Guajira. Sin embargo, la limitada información de distribución y la ausencia de datos poblacionales, dificultan establecer con precisión las áreas frecuentadas por esta especie y delimitar adecuadamente reservas para su conservación a escala regional que puedan contribuir a la conservación de la población de osos andinos al norte de Colombia. El desconocimiento 
de la distribución de T. ornatus pone en riesgo la sobrevivencia de sus poblaciones locales (Kattan et al. 2004). Con base en nuestros hallazgos, la reciente declaración del Parque Natural Regional (PNR) Cerro Pintao (Corpoguajira 2016) podría estar excluyendo áreas usadas por $T$. ornatus (Figura 2). Aunque se sabe que $T$. ornatus prefiere los bosques andinos como hábitat, esta especie también puede usar los paisajes agrícolas de partes altas como sitios de alimentación o tránsito (Goldstein et al. 2006). Los avistamientos que se hicieron por fuera del área protegida coinciden con una zona transformada en la que se ha perdido y fragmentado parte del bosque nativo (Figura 2). Confirmar que estos sitios de avistamiento representan ambientes frecuentados por esta especie requerirá más información de campo, y de esta manera posiblemente se contribuya a la redefinición de los límites del PNR. Por lo tanto, se sugiere seguir adelantando esfuerzos para aumentar el conocimiento local de la distribución de T. ornatus al sur del departamento de La Guajira y así generar áreas de protección que incluyan todas las zonas de ocurrencia de esta especie.

\section{AGRADECIMIENTOS}

A C. Pérez por el apoyo logístico durante la expedición. A L. Morelos por su ayuda en la identificación de las semillas. A los pobladores locales por compartir sus experiencias de encuentros con el Oso. Finalmente, a los revisores por mejorar el documento con sus observaciones.

\section{REFERENCIAS}

Cavelier J, Lizcano DJ, Yerena E, Downer C. 2010. The mountain tapir (Tapirus pinchaque) and Andean bear (Tremarctos ornatus): two charismatic, large mammals in South America tropical montane cloud forests, In: Bruijinzeel LA, Scatena, FN, Hamilton LS, editors. Tropical Mountain Cloud Forest: Science for Conservation and Management. Cambridge University Press, p. 172-181.

Corpoguajira. 2016. Acuerdo del Consejo Directivo No. 032 del 22 de diciembre de 2016.

García-Rangel S. 2012. Andean bear Tremarctos ornatus natural history and conservation. Mammal Review 42:85-119. https:// doi.org/10.1111/j.1365-2907.2011.00207.x

Goldstein I, Paisley S, Wallace R, Jorgenson J, Cuesta F, Castellanos A. 2006. Andean bear-livestock conflicts: a review. Ursus 17:8-15. https:// doi.org/10.2192/1537-6176(2006)17[8:ABCAR]2.0.CO;2

Kattan G, Hernández OL, Goldstein I, Rojas V, Murillo O, Gómez C, Restrepo H, Cuesta F. 2004. Range fragmentation in the spectacled bear Tremarctos ornatus in the northern Andes. Oryx 38:155-163. https://doi.org/10.1017/S0030605304000298

Mondolfi E. 1989. Notes on the distribution, habitat, food habits, status and conservation of the Spectacled bear (Tremarctos ornatus Cuvier) in Venezuela. Mammalia 53:525-544. https://doi.org/https://doi.org/10.1515/mamm.1989.53.4.525

Rodríguez-Castro D, Contento S, Grajales D, Rodríguez D. 2015. Evaluación del estado de aplicación del Programa Nacional para la Conservación en Colombia del Oso Andino (Tremarctos ornatus). Revista Biodiversidad Neotropical 5:36-46.

Rodríguez D, Reyes A, Contreras P, Hernandez G. 2016. Implementación del plan de acción regional para la conservación del oso andino (Tremarctos ornatus) en la eco región Serranía del Perijá, en el marco del plan nacional de conservación Oso Andino (segunda fase). Informe final. Corpocesar-Fundación Wii. 1-72. 
Rodríguez D, Reyes A, Reyes-Amaya N, Gallegos-Sánchez S, Gutierrez J, Suárez R, Prieto F. 2019. Northernmost distribution of the Andean bear (Tremarctos ornatus) in South America, and fragmentation of its associated Andean forest and paramo ecosystems. Therya 10:161-170. https:// doi.org/10.12933/therya-19-756

Rodríguez D, Reyes A, Rincon S, Medina T, Medina J, Mirith M, Galindo M, Reyes N. 2011. Implementación del plan de acción regional para la conservación del oso andino (Tremarctos ornatus) en la eco región serranía del Perijá, en marco del programa nacional conservación de oso andino. Informe final. Corpocesar-Fundación Wii. 1-304.

Rodríguez D, Reyes A, Rincon S, Reyes N, Contreras P, Hernández G. 2014. Implementación del plan de acción regional para la conservación del oso andino (Tremarctos ornatus) en la eco región serranía del Perijá, en marco del programa nacional conservación de oso andino. Informe final. Corpocesar-Fundación Wii. 1-191.

Velez-Liendo X, García-Rangel S. 2017. Tremarctos ornatus, Spectacled Bear (errata version published in 2018). IUCN Red List Threat. Species 8235. https://dx.doi.org/10.2305/IUCN.UK.20173.RLTS.T22066A45034047.en. (Consultado: 28/03/2020).

Viloria A, Mondolfi E, Yerena E, Herrera F. 1995. Nuevos registros del oso de anteojos o frontino (Tremarctos ornatus, F. Cuvier) en la sierra de Perijá Venezuela. Sociedad de Ciencias Naturales La Salle Tomo LV 143:1-11.

Editor: Daniel Rodríguez Recibido: 2020-03-23

Revisado: 2020-04-06

Aceptado: 2020-05-10

Publicado 2020-05-25 